\title{
SPOCK2 Affects the Biological Behavior of Endometrial Cancer Cells by Regulation of MTI-MMP and MMP2
}

Reproductive Sciences

|39|-1399

(C) The Author(s) 2019

DOI: 10.1007/s43032-020-00197-4

\author{
Fang Ren, PhD', Danni Wang, PhD', Yizi Wang, MD', \\ Peng Chen, PhD' $^{\prime}$, and Cuishan Guo, PhD' $^{\prime}$
}

\begin{abstract}
Abnormal expression of SPARC (osteonectin), cwcv and kazal-like domains proteoglycan 2 (SPOCK2) plays a significant role in the development and progression of various human cancers, yet a relationship between SPOCK2 and endometrial cancer (EC) has not been reported. Here, we assessed the potential role and mechanism by which SPOCK2 acts in the pathogenesis and progression of EC. First, protein expression of SPOCK2 in EC tissue from patients was detected by immunohistochemistry and associated clinical data were analyzed. Then, HEC-IA and Ishikawa cells were transfected with an adenoviral vector containing an SPOCK2 recombinant fragment and the biological behavior of transfected cells was observed. Finally, the expression of membrane type I matrix metalloproteinase (MTI-MMP) and MMP2 in the transfected cells was detected by Western blot and zymography gel assay to analyze the effect of SPOCK2 on the regulation of the MTI-MMP/MMP2 pathway. We found that there was significantly less SPOCK2 protein expression in the EC tissue than in the normal endometrium tissue, and lack of SPOCK2 protein expression in EC tissue was associated with distant metastasis and myometrial invasion. Upregulation of SPOCK2 in HECIA and Ishikawa cells inhibited cell proliferation, invasion, adhesion, and apoptosis. Upregulation of SPOCK2 inhibited the expression of MTI-MMP and MMP2 and activation of MMP2 in HEC-IA and Ishikawa cells. Collectively, our data indicated that SPOCK2 contributed to the progression of EC by regulating the biological behavior of cancer cells, which is achieved partly through regulating protein expression of MTI-MMP and MMP2 and activation of MMP2.
\end{abstract}

\section{Keywords}

SPOCK2, endometrial cancer, MTI-MMP, MMP2, cell proliferation, invasion, adhesion, apoptosis

\section{Introduction}

Endometrial cancer (EC) is the most common female reproductive malignancy in developed countries and is the second most common in China. The incidence is 63.4 in 100000 and the mortality rate is 21.8 of 100000 in China, according to the statistics reported by the National Cancer Center of China in 2015. Currently, the incidence of EC is increasing worldwide; this trend is mainly ascribed to the increasing incidence of obesity and nulliparity. ${ }^{1}$ According to its relationship with estrogen and progesterone as well as epidemiological and prognostic factors, EC is divided into 2 types. ${ }^{2}$ Type I EC is estrogen dependent and occurs in proliferative endometrium, which accounts for $80 \%$ of all ECs. Precancerous lesions of type I EC are termed endometrial atypical hyperplasia, while its pathological type is endometrioid adenocarcinoma. Endometrial cancer of this type presents at an early stage and the patient has a good prognosis. Type II is not estrogen sensitive and often occurs in the presence of atrophic endometrium. The lesion is high grade and patients of this type may have a poor prognosis. Endometrial cancers of different types may also differ in pathogenesis. $^{3}$
SPARC (osteonectin), cwcv and kazal-like domains proteoglycan 2 (SPOCK2), also known as testincan-2, is a member of the SPOCK family. This gene encodes a protein that binds to glycosaminoglycans to form part of the extracellular matrix (ECM). The protein contains thyroglobulin type 1, follistatinlike, and calcium-binding domains and has glycosaminoglycan attachment sites in the acidic C-terminal region. It is widely expressed in various tissues. In 2008, a study found that the oligonucleotide polymorphism of SPOCK2 gene was related to the deletion of $16 \mathrm{q}$ chromosome in breast cancer ${ }^{4}$; this was the first report to describe the correlation between the SPOCK2 gene and cancer. Then Chung et $\mathrm{al}^{5}$ found that abnormal methylation of SPOCK2 gene was closely associated with

\footnotetext{
' Department of Obstetrics and Gynecology, Shengjing Hospital of China Medical University, Shenyang, Liaoning, China

Corresponding Author:

Fang Ren, Department of Obstetrics and Gynecology, Shengjing Hospital of China Medical University, 36 Sanhao Street, Shenyang, Liaoning I I0004, China. Email: renfang006329@I63.com
} 
prostate cancer, breast cancer, and colon cancer; thus, screening for epigenetic inactivation of SPOCK2 could be used for early diagnosis of these 3 tumors. In our previous study, ${ }^{6}$ we screened for genes related to malignant transformation of ovarian endometriosis by methylation $\mathrm{CpG}$ island amplification representational difference analysis; we found that SPOCK2 protein was absent in endometriosis-related ovarian endometrioid adenocarcinoma because of gene hypermethylation, indicating that the absence of SPOCK2 may be related to the tumorigenesis and progress of endometriosis-related ovarian endometrioid adenocarcinoma. Endometriosis-related ovarian endometrioid adenocarcinoma and type I uterine endometrioid adenocarcinoma are related in origin and share similar highrisk factors, such as high estrogen stimulation ${ }^{7}$; furthermore, uterine endometrioid adenocarcinoma is commonly found concomitantly with ovarian endometrial adenocarcinoma. ${ }^{8}$ Thus, we speculated that ovarian endometrioid cancer-related genes (such as SPOCK2) might also play an important role in the tumorigenesis and progression of type I uterine endometrioid adenocarcinoma. However, the relationship between SPOCK2 and uterine endometrioid adenocarcinoma is still unknown. Here, we investigated the role of the SPOCK2 gene in the uterine endometrioid adenocarcinoma to begin to explore its role and mechanism in the progression of endometrial adenocarcinoma.

\section{Materials and Methods}

\section{Detection of SPOCK2 Protein Expression in Endometrial Adenocarcinoma by Immunohistochemistry}

All specimens were obtained from patients hospitalized in the Department of Obstetrics and Gynecology of Shengjing Hospital (Shenyang, China). For the endometrioid adenocarcinoma (EC) group, 30 formalin-fixed paraffin-embedded (FFPE) tissue specimens of type I endometrioid adenocarcinoma were obtained from patients who were diagnosed between 2015 and 2016. For the normal endometrium (NE) group, 20 FFPE tissue specimens of normal secretory phase endometrium were obtained from patients who suffered cervical intraepithelial neoplasia III and underwent hysterectomy. Informed consent was obtained from all patients or their family members, and agreement was obtained from the ethics committee of China Medical University before collecting the samples. The mean age of the patients in the 2 groups was 44.19 (9.86) and 43.52 (5.16) years, respectively; there was no statistical difference in age between the 2 groups $(P>.05)$. None of the patients had received any gonadotropin-releasing hormone analog or other hormonal medication nor any antibiotics in the 6 months prior to the surgery.

The FFPE specimens were cut into $4-\mu \mathrm{m}$ sections. The protein expression of SPOCK2 was evaluated by immunohistochemistry, which was performed with a standard streptavidin-peroxidase technique using diaminobenzidine as a chromogen. Goat polyclonal antibody against SPOCK2 (dilution 1:200; Santa Cruz, California, USA) was used as the
Table I. Primers of SPOCK2 for Real-Time PCR.

\begin{tabular}{|c|c|c|c|}
\hline Name & Sequence & $T_{\mathrm{m}}$ & $\begin{array}{l}\text { Product } \\
\text { Length }\end{array}$ \\
\hline SPOCK2 F & ATGGAGGACGAGCAATGGCT & 61.8 & 93 \\
\hline SPOCK2 R & TGGGTCGGACGAGGGAAC & 60.8 & \\
\hline$\beta$-Actin F & CTTAGTTGCGTTACACCCTTTCTTG & 62 & 156 \\
\hline$\beta$-Actin R & CTGTCACCTTCACCGTTCCAGTTT & 64.4 & \\
\hline
\end{tabular}

Abbreviations: PCR, polymerase chain reaction; SPOCK2, SPARC

(osteonectin), cwcv and kazal-like domains proteoglycan 2.

primary antibody; phosphate-buffered saline was used as the negative control. The normal staining pattern for SPOCK2 is cytoplasmic. The percentage of cells positive for SPOCK2 expression was evaluated by counting at least 5 different areas of the sections at high magnification $(\times 1000)$. The immunoreactivity was determined by 2 independent observers.

\section{Cell Culture and Transfection}

HEC-1A cells and Ishikawa cells were purchased from the Shanghai Institute of Cell Biology of the Chinese Academy of Sciences (Shanghai, China). The cells were cultured in Dulbecco's modified Eagle medium high glucose media and RPMI-1640 supplemented 10\% fetal bovine serum (FBS), respectively, in a sterile incubator at $37^{\circ} \mathrm{C}$ under $5 \% \mathrm{CO}_{2}$.

The SPOCK 2 recombinant fragment and the adenoviral vector were purchased from GenePharma (Shanghai, China). The transfection was performed according to the manufacturer's instructions. Mock transfected cells were used as negative controls; cells transfected with empty vector were used as vector controls. At 48 hours posttransfection, the supernatant was discarded and replaced with complete medium; then cells were cultured for another 24 hours at $37^{\circ} \mathrm{C}$ under $5 \% \mathrm{CO}_{2}$ for further study.

\section{Detection of SPOCK2 Messenger RNA Expression in Cells by Real-Time Polymerase Chain Reaction}

Total RNA was obtained from cells using TRIzol reagent. After RNA was quantified, the expression of SPOCK2 messenger RNA (mRNA) in cells was detected by real-time polymerase chain reaction (PCR). The primer information is shown in Table 1. The reaction conditions were as follows: incubation at $94^{\circ} \mathrm{C}$ for 5 minutes, 40 cycles of incubation at $94^{\circ} \mathrm{C}$ for 10 seconds, $60^{\circ} \mathrm{C}$ for 20 seconds, and $72^{\circ} \mathrm{C}$ for 30 seconds. Data were analyzed using the $2^{-\triangle \triangle \mathrm{CT}}$ method. All reactions were carried out under RNAase-free conditions, and each sample was assayed in triplicate.

\section{Detection of SPOCK2 Protein Expression in Cells by Western Blot}

HEC-1A and Ishikawa cells were harvested at 48 hours after transfection. Radioimmunoprecipitation assay buffer (Keygen Biotech, Beijing, China) containing protease inhibitors and 
phosphatase inhibitors was added to the samples; then, the samples were placed on ice for 5 minutes; samples were then centrifuged at $12000 \mathrm{rpm}, 4^{\circ} \mathrm{C}$ for 10 minutes, and the supernatant was removed to obtain total protein. After the total protein was quantified, sodium dodecyl sulfate polyacrylamide gel electrophoresis (SDS-PAGE) was carried out at $80 \mathrm{~V}$ for 2.5 hours. The loading volume of this experiment was $20 \mu \mathrm{L}$, containing $40 \mu \mathrm{g}$ of protein. After being blocked for 1 hour with $5 \%$ skim milk, the membranes were incubated with specific primary antibodies overnight at $4{ }^{\circ} \mathrm{C}$, followed by secondary antibodies for 2 hours at room temperature. The anti-POCK2 polyclonal antibody (1:200) was purchased from Santa Cruz, antimembrane-type 1 matrix metalloproteinase (anti-MT1-MMP; 1:1000) from Proteintech (Wuhan, China), and anti-MMP2 (1:500) from Wanleibio (Shenyang, China). Secondary antibody (1:5000) was purchased from Wanleibio. The film was scanned and the optical density (OD) value of the target strip was analyzed using a gel image processing system (Gel-Pro-Analyzer software version 4.0). All assays were performed in triplicate.

\section{Detection of the Cell Proliferation Rate Using a Cell Counting Kit-8}

HEC-1A and Ishikawa cells were seeded into 96-well plates, each group was seeded to 5 replicate wells at $4 \times 10^{3}$ cells/ well. After transfection for $24,48,72$, or 96 hours, $10 \mu \mathrm{L}$ Cell Counting Kit-8 (CCK8) reagent was added to each well. Cells were incubated for another 3 hours in a $37^{\circ} \mathrm{C}$ under $5 \% \mathrm{CO}_{2}$ before measuring the $\mathrm{OD}$ at $450 \mathrm{~nm}$ with an enzyme-linked immunosorbent assay plate reader (Bio-Rad). All assays were performed in triplicate.

\section{Detection of Cell Cycle Using Flow Cytometry}

Transfected HEC-1A and Ishikawa cells were fixed with precooled $70 \%$ ethanol for 2 hours at $4^{\circ} \mathrm{C}$. Then $100 \mu \mathrm{L}$ RNase A were added to fixed cells and incubated at $37^{\circ} \mathrm{C}$ for 30 minutes; cells were then stained with $500 \mu \mathrm{L}$ propidium iodide for another 30 minutes. DNA content was assayed with a flow cytometer and analyzed using ModFit LT software version 3.0. All assays were performed in triplicate.

\section{Detection of Cell Invasion by Transwell Chamber}

Matrigel gel was thawed on ice and diluted with serum-free medium. The transwell chamber was coated by $40 \mu \mathrm{L}$ prediluted Matrigel gel and placed in a $37^{\circ} \mathrm{C}$ incubator for 2 hours to solidify. The Matrigel-coated transwell chamber was placed in a 24-well plate and $800 \mu \mathrm{L}$ of medium containing 30\% FBS was added to the lower chamber, while $200 \mu \mathrm{L}$ of the cell suspension $\left(2 \times 10^{4}\right)$ was added to the upper chamber. The 24-well plates were incubated at $37^{\circ} \mathrm{C}$ under $5 \% \mathrm{CO}_{2}$ for 26 hours at which time cells could be seen in the lower chamber. Cells remaining on the top of the transwell chamber were scraped off with a cotton swab. Invasive cells were fixed with $4 \%$ paraformaldehyde for 20 minutes and stained with $0.5 \%$ crystal violet for 5 minutes; cells in the lower layer of the membrane were counted under an inverted microscope $(\times 200)$.

\section{Detection of the Cell Adhesion Ability Using a Cell Adhesion Assay}

Transfected HEC-1A and Ishikawa cells were inoculated into 96-well plates that were coated with Matrigel or bovine serum albumin (BSA) at the same density $\left(1 \times 10^{4}\right)$ and cultured for 2 hours; then $0.5 \mathrm{mg} / \mathrm{mL}$ of 3-(4,5-Dimethylthiazol-2-yl)-2,5diphenyltetrazolium bromide was added to the plates for 4 hours. After removing the supernatant, $200 \mu \mathrm{L}$ of dimethyl sulfoxide was added to dissolve the crystal formed by the cells, and the $\mathrm{OD}$ at $570 \mathrm{~nm}$ was measured on a microplate reader. Adhesion rate $=$ (mean OD value of the Matrigel-coated well $/$ mean OD value of BSA-coated well -1$) \times 100 \%$.

\section{Detection of Cell Apoptosis by Flow Cytometry}

Transfected HEC-1A and Ishikawa cells were mixed with 500 $\mu \mathrm{L}$ of binding buffer; then $5 \mu \mathrm{L}$ of annexin $\mathrm{V}$-allophycocyanin and $5 \mu \mathrm{L}$ of propidium iodide were added to the cells to incubate for 15 minutes at room temperature in the dark. DNA content was assayed by a flow cytometer and analyzed using ModFit LT software. All assays were performed in triplicate.

\section{Detection of MMP2 Expression in Cells by Zymography Gel Assay}

The cell supernatant was obtained after centrifugation. After measuring the concentration, $60 \mu \mathrm{g}$ of protein was used for SDS-PAGE with constant voltage overnight, and the gel was stained in the staining solution for 3 hours. After being decolorized, the gel was photographed by a gel imaging system.

\section{Statistical Analysis}

All data were expressed as the mean (standard deviation). SPSS 13.0 software was used for the statistical tests. A Student $t$ test was used to determine the significance of 2 group differences. Chi-square tests were used to compare SPOCK2 protein expression and clinicopathological features. A $P<.05$ (2tailed) was considered to be significant.

\section{Results}

\section{The SPOCK2 Protein Expression in Different Groups}

The expression of SPOCK2 protein in tissues from each group was detected using immunohistochemistry. The result is shown in Table 2 and Figure 1. The rate of SPOCK2 protein absence in the EC group was $53.33 \%$, which was significantly higher than that in the NE group. There was a significant statistical difference between the 2 groups $(P<.05)$. After further analysis, the clinical data from the EC group revealed that the absence of SPOCK2 protein expression in cancer tissue was related to distant metastases and myometrial invasion $(P<.05)$, but not to cancer stage or age of patient $(P>.05)$. 
Table 2. Expression of SPOCK2 Protein in Each Group.

\begin{tabular}{|c|c|c|c|c|}
\hline \multirow[b]{2}{*}{ Group } & \multirow[b]{2}{*}{ Number } & \multicolumn{2}{|c|}{$\begin{array}{l}\text { SPOCK2 Protein } \\
\text { Expression }\end{array}$} & \multirow[b]{2}{*}{$P$ Value } \\
\hline & & Positive & Negative & \\
\hline Endometrial cancer & 30 & 14 & 16 & \\
\hline Age (years) & & & & .796 \\
\hline$<50$ & 17 & 5 & 5 & \\
\hline$\geqq 50$ & 13 & 9 & II & \\
\hline FIGO stages & & & & .272 \\
\hline I-II & 24 & 10 & 14 & \\
\hline III-IV & 6 & 4 & 2 & \\
\hline Myometrial invasion & & & & .007 \\
\hline$<1 / 2$ & 8 & 7 & I & \\
\hline$\geqq 1 / 2$ & 22 & 7 & 15 & \\
\hline Lymphatic metastasis & & & & .024 \\
\hline No & 22 & 13 & 9 & \\
\hline Yes & 8 & 1 & 7 & \\
\hline Normal endometrium & 20 & 18 & 2 & .002 \\
\hline
\end{tabular}

Abbreviation: SPOCK2, SPARC (osteonectin), cwcv and kazal-like domains proteoglycan 2.

\section{The SPOCK2 Gene Expression After Transfection}

Both mRNA and protein expression of SPOCK2 in HEC-1A and Ishikawa cells were assayed using real-time PCR and
Western blotting after transfection. The results are shown in Figure 2. The SPOCK2 mRNA and protein expression was significantly increased in cells after being transfected and the difference was statistically significant $(P<.05)$. The results indicate that transfection can increase the expression of SPOCK2 effectively in HEC-1A and Ishikawa cells.

\section{Cellular Proliferation After SPOCK2 Upregulation}

The proliferation of HEC-1A and Ishikawa cells after transfection was assayed using a CCK8. Results are shown in Figure 3. The cell proliferation rate was significantly upregulated 24 hours after transfection; the cell proliferation rate of the transfected group was significantly lower than that of the negative control group and the vector control group and the difference was statistically significant $(P<.05)$. These results indicate that upregulation of SPOCK2 can inhibit the proliferation of HEC-1A and Ishikawa cells.

\section{Cell Cycle Changes After SPOCK2 Upregulation}

Cell cycle analysis of HEC-1A and Ishikawa cells after transfection was performed using flow cytometry. Results are shown in Figure 4. The percentage of cells in G0/G1 phase increased and the percentage of cells in $\mathrm{S}$ phase decreased in

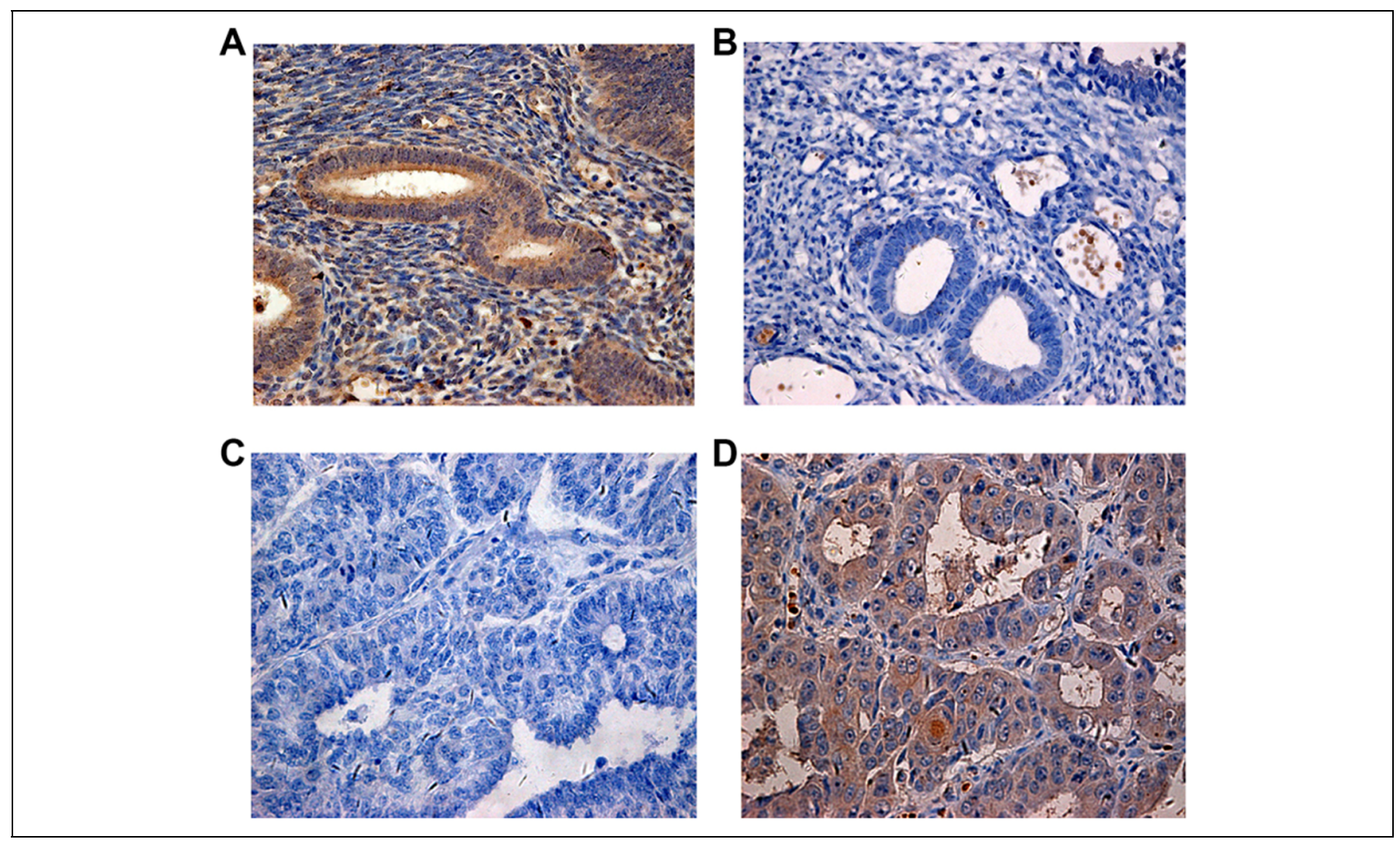

Figure I. Detection of SPOCK2 protein expression in tissues by immunohistochemistry. A, Normal SPOCK2 protein expression in NE. B, Absence of SPOCK 2 protein expression in NE. C, Absence of SPOCK2 protein expression in EC. D, Normal SPOCK 2 protein expression in EC. Magnification $\times 200$. EC indicates endometrial cancer; NE, normal endometrium; SPOCK2, SPARC (osteonectin), cwcv and kazal-like domains proteoglycan 2. 


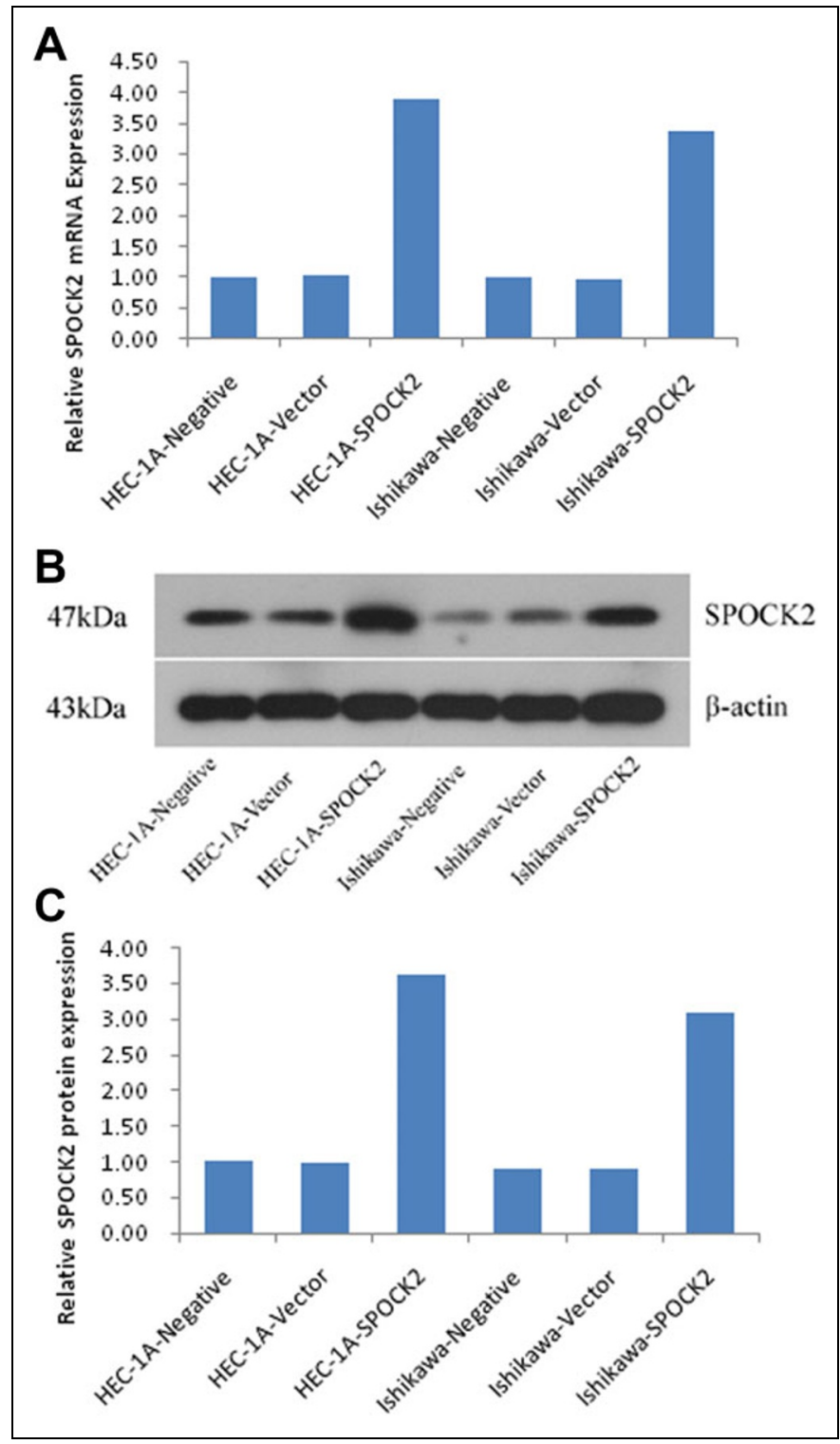

Figure 2. Expression of SPOCK2 in HEC-IA and Ishikawa cells after transfection. A, The SPOCK2 mRNA expression in cells was measured using real-time PCR. The mRNA expression of SPOCK2 in cells increased after transfection $(P<.05)$. B and $C$, The SPOCK2 protein expression in cells was detected via Western blot. The protein expression of SPOCK 2 in cells increased after transfection $(P<.05)$. mRNA indicates messenger RNA; PCR, polymerase chain reaction; SPOCK2, SPARC (osteonectin), cwCV and kazal-like domains proteoglycan 2.

the transfection group as compared to the negative control group and the vector control group $(P<.05)$. These results indicate that upregulation of SPOCK2 can inhibit cell cycle progression in HEC-1A and Ishikawa cells.

\section{Invasive Ability After SPOCK2 Upregulation}

The invasive ability of HEC-1A and Ishikawa cells transfected was analyzed using a Transwell invasion chamber. Results are shown in Figure 5. The invasive ability of the transfected group

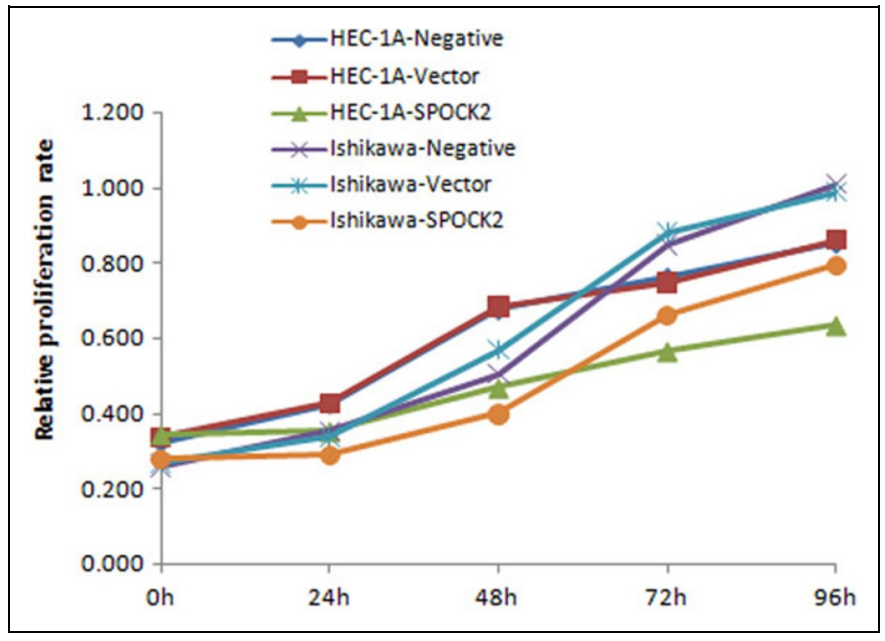

Figure 3. Influence of SPOCK2 on cell proliferation in HEC-IA and Ishikawa cells transfected with SPOCK2. Proliferation of Ishikawa and HEC-IA cells was significantly decreased after SPOCK2 upregulation $(P<.05)$. SPOCK 2 indicates SPARC (osteonectin), cwcv and kazal-like domains proteoglycan 2.

was significantly lower than that of the negative control group and vector control group. The difference was statistically significant $(P<.05)$. These results indicate that upregulation of SPOCK 2 can inhibit the invasive ability of HEC-1A and Ishikawa cells.

\section{Cellular Adherence After SPOCK2 Upregulation}

Adherence in HEC-1A and Ishikawa cells after transfection was measured using a Matrigel adhesion assay. Results are shown in Figure 6. Adherence of cells in the transfected group was significantly lower than that in the negative control group and the vector control group, and the difference was statistically significant $(P<.05)$. These results indicate that upregulation of SPOCK2 can inhibit adherence in HEC-1A and Ishikawa cells.

\section{Apoptosis After SPOCK2 Upregulation}

Apoptosis of HEC-1A and Ishikawa cells after transection was detected using annexin V-Fluorescein isothiocyanate/Propidium iodide staining followed by analysis using flow cytometry. Results are shown in Figure 7. The rate of apoptosis in the transfected group was significantly higher than that of the negative control group and vector control group. The difference was statistically significant $(P<.05)$. These results indicate that upregulation of SPOCK2 can promote apoptosis of HEC-1A and Ishikawa cells.

\section{The MTI-MMP2 and MMP2 Protein Expression and the Expression of Active MMP2 Protein in Cells After SPOCK2 Upregulation}

The expression of MT1-MMP2 and MMP2 protein in HEC-1A and Ishikawa cells after transfection was detected by Western 


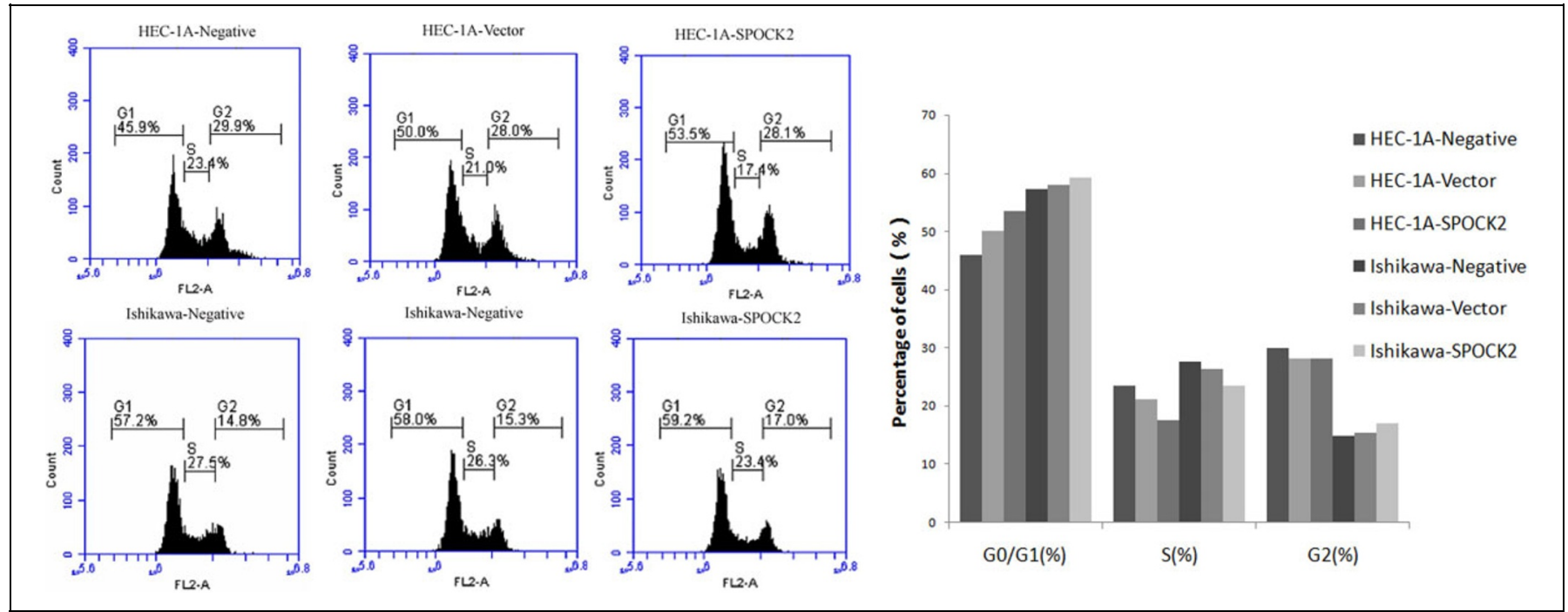

Figure 4. Influence of SPOCK2 on the cell cycle in HEC-IA and Ishikawa cells transfected with SPOCK2. The percentage cells in G0/GI phase increased $(P<.05)$ and the percentage cells in $S$ phase decreased significantly after SPOCK2 upregulation $(P<.05)$. SPOCK2 indicates SPARC (osteonectin), cwcr and kazal-like domains proteoglycan 2.

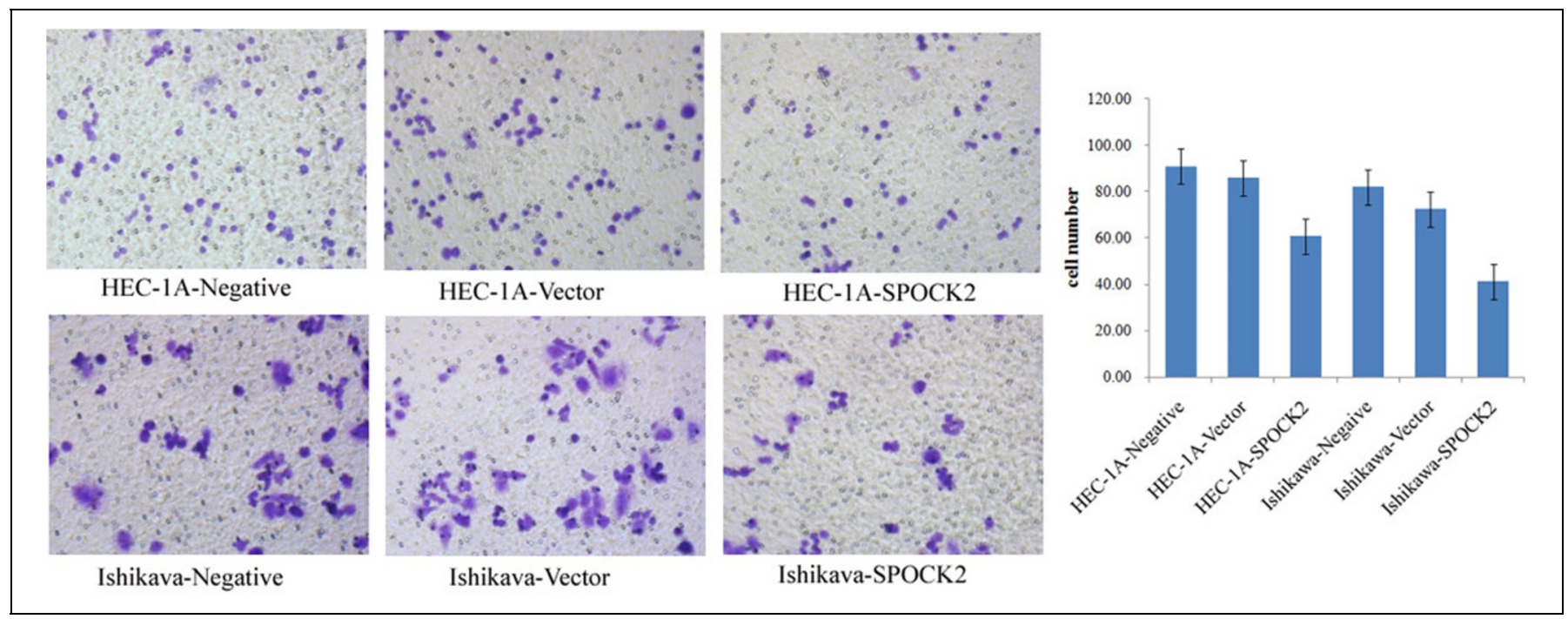

Figure 5. Influence of SPOCK2 on invasive ability in HEC-IA and Ishikawa cells transfected with SPOCK2. The number of Ishikawa and HEC-IA cells migrating into the lower chamber was significantly reduced after SPOCK2 upregulation $(P<.05)$. SPOCK2 indicates SPARC (osteonectin), cwcv and kazal-like domains proteoglycan 2.

blot. Results are shown in Figure 8. The expression of MT1MMP2 and MMP2 in cells in the transfected group was significantly lower than those in the negative control group and the vector control group, and the difference was statistically significant $(P<.05)$. The results indicate that upregulation of SPOCK2 can inhibit the expression of MT1-MMP2 and MMP2 in HEC-1A and Ishikawa cells.

The expression of activated MMP2 was detected using gelatin zymography after transfection. Expression of activated MMP2 protein after transfection was significantly lower than in the negative control group or the vector control group, and the difference was statistically significant $(P<.05)$. These results indicate that upregulation of SPOCK2 can inhibit MMP2 protein activation.

\section{Discussion}

The SPOCK is a glycoprotein composed of a protein domain, 2 chondroitin side chains, and heparan sulfate. The protein domain contains adhesion/antiadhesion molecules, growth factor-binding proteins, and protease inhibitors. ${ }^{9}$ Sambuudash et $\mathrm{al}^{10}$ have shown that hypermethylation of the SPOCK2 gene in colon cancer is significantly higher than that in the normal mucosal tissue adjacent to the cancer. We also previously 


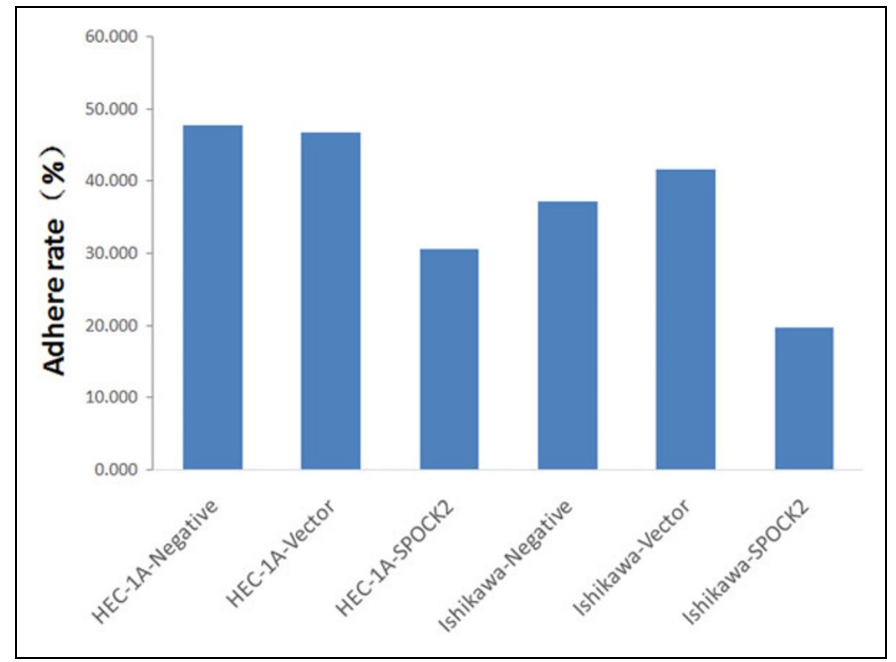

Figure 6. Influence of SPOCK2 on adherence by HEC-IA and Ishikawa cells transfected with SPOCK2. The adhesion rate of HEC-IA and Ishikawa cells decreased significantly after SPOCK2 upregulation $(P<.05)$. SPOCK2 indicates SPARC (osteonectin), cwcv and kazal-like domains proteoglycan 2 .

reported that hypermethylation of the SPOCK2 gene was associated with endometriosis-related ovarian endometrioid adenocarcinoma, ${ }^{11}$ indicating that abnormalities in this gene might contribute to tumorigenesis and the progression of tumors. In this study, we measured protein expression of SPOCK2 in type I EC and found that the expression of SPOCK2 protein in EC tissues was significantly lower than that in the NE. After further analyzing the clinical data of patients with $\mathrm{EC}$, we found that the loss of SPOCK2 protein expression was related to the infiltration of the basal layer and distant metastasis, but not to International Federation of Gynecology and Obstetrics stage or age of the patients. These results indicate that loss of SPOCK2 protein expression might be involved in the progression, and especially in the invasion and metastasis of EC. To study the role of the SPOCK2 gene in the progression of EC, we ectopically expressed the $S P O C K 2$ gene in moderately differentiated (HEC-1A) and highly differentiated (Ishikawa) EC cells and observed its effect on the biological activity of the transfected cells. Our results indicate that upregulation of SPOCK2 in EC cells can inhibit cell proliferation, invasion, adhesion, and apoptosis, thus making SPOCK2 a potential target for the EC therapy. Since SPOCK2 gene was commonly found to be inactivated by promoter methylation, which is a reversible epigenetic change, the upregulation of SPOCK2 by reversing the methylation of this gene could be used for EC treatment to promote better disease outcome.

Metalloproteinase 2 is a type of collagenase that breaks down plasma fibronectin and laminin in the basement membrane. It is involved in various physiological and pathological processes in the human body, such as angiogenesis, tumor growth, tumor invasion, and metastasis. ${ }^{12,13}$ The MMP2 is regulated by various cell signaling pathways during transcription and by proteins during its activation from proproteinase to enzyme. ${ }^{14}$ Membrane-type 1 matrix metalloproteinase is a member of the MMP family. It can specifically regulate the activation of MMP2 and bind to activated MMP2, which is associated with malignant biological behaviors in a variety of tumor types. ${ }^{15,16}$ The MT1-MMP also displays a broad spectrum of activities against ECM components by itself as various cell types employ MT1-MMP to alter their surrounding environment during angiogenesis, tissue remodeling, tumor invasion, and metastasis. ${ }^{17,18}$ In a study of glioma, it was found that SPOCK1 and SPOCK3 could inhibit MT1-MMP-mediated MMP2 activation and inhibit glioma cell invasion by binding

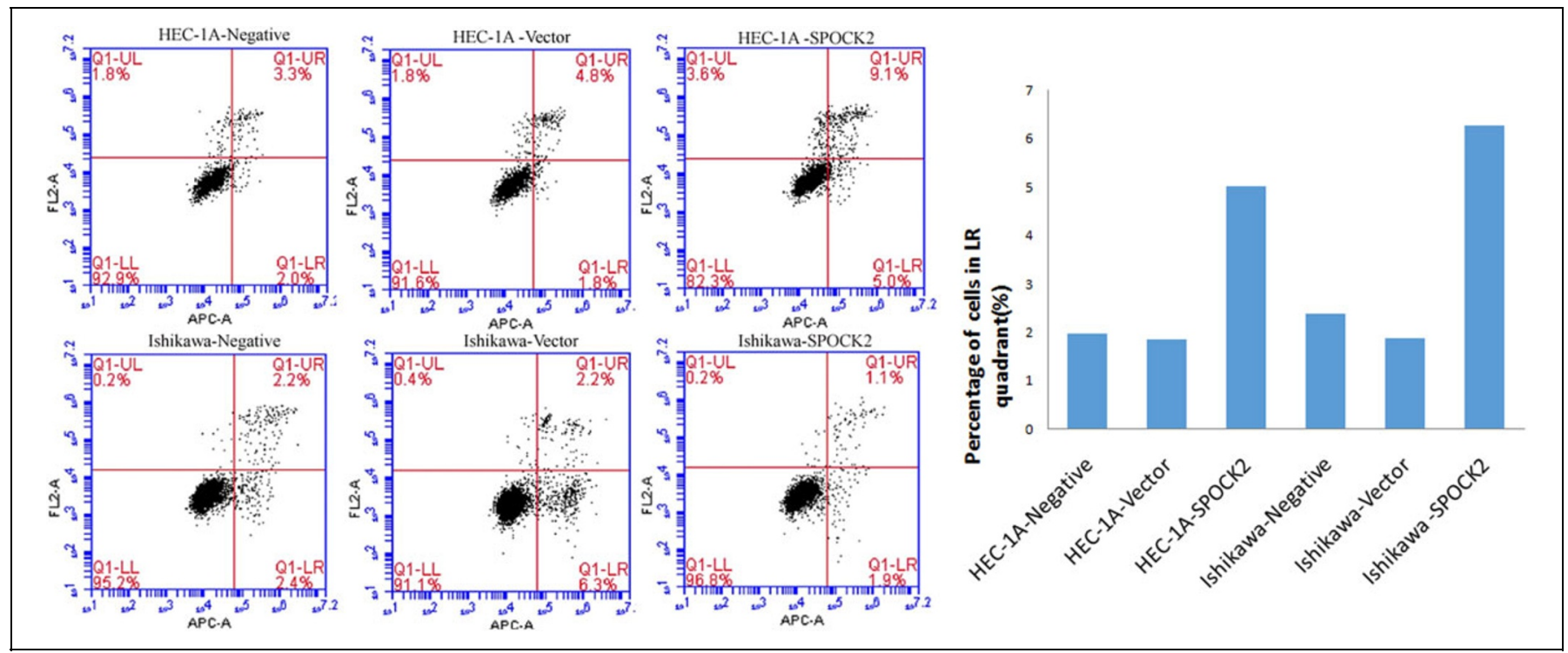

Figure 7. Influence of SPOCK2 on apoptosis after HEC-IA and Ishikawa cells were transfected with SPOCK2. The number of HEC-IA and Ishikawa cells undergoing apoptosis decreased significantly after SPOCK2 upregulation $(P<.05)$. SPOCK2 indicates SPARC (osteonectin), cwcr and kazal-like domains proteoglycan 2. 

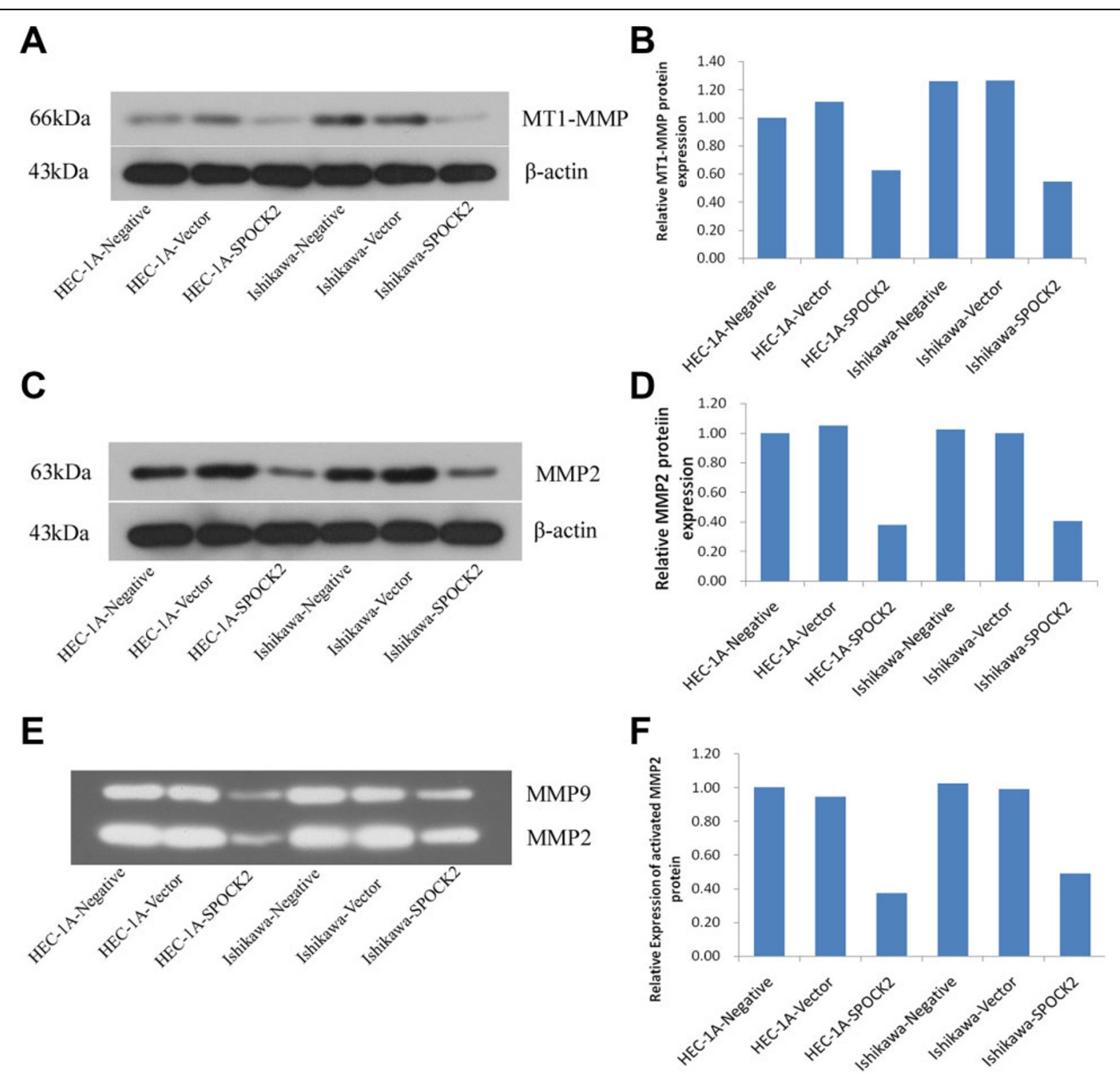

Figure 8. Influence of SPOCK2 upregulation on the MTI-MMP/MMP2 pathway. A, Expression of MTI-MMP2 protein in HEC-IA and Ishikawa cells transfected with SPOCK2 was detected via Western blot. The MTI-MMP protein expression decreased after SPOCK2 upregulation $(P<$ .05). B, Expression of MMP2 in HEC-IA and Ishikawa cells after transfection with SPOCK2 was detected via Western blot. The MMP2 protein expression decreased after SPOCK2 upregulation $(P<.05)$. C, Expression of activated MMP2 after transfection with SPOCK2 was analyzed using gelatin zymography. Activated MMP2 decreased after SPOCK2 upregulation $(P<.05)$. MTI-MMP indicates membrane-type I matrix metalloproteinase; MMP2, metalloproteinase 2; SPOCK2, SPARC (osteonectin), cwcv and kazal-like domains proteoglycan 2.

to MT1-MMP ${ }^{19}$; furthermore, high-level expression of SPOCK2 could abrogate the inhibition of MMP2 by SPOCK1 and SPOCK3 and increase the invasiveness of glioma cells. ${ }^{20}$ However, another study on the mouse model of Pseudomonas aeruginosa-induced keratitis indicated that SPOCK1 upregulation could increase MMP2 expression and activation and SPOCK1 downregulation could inhibit MMP2 expression and activation. ${ }^{21}$ To explore the mechanism by which SPOCK2 influences the biological behavior of EC cells, we further examined MT1-MMP and MMP2 expression and MMP2 activation in EC cells after SPOCK2 upregulation. In contrast to the results reported by Nakada et al, our data indicated that upregulation of SPOCK2 in EC cells could decrease MMP2 activation and inhibit cell invasion. The mechanism of the inhibiting MMP2 by SPOCK2 was unclear, and we speculate
2 possibilities: (1) It was studied in the glioma cell that SPOCK family members can bind directly with MT1-MMP and affect the MT1-MMP-mediated MMP2 activation, while with the regulation of the other MMPs, such as TIMP $1,{ }^{22}$ upregulation of SPOCK2 in EC may decrease MMP2 activation by inhibiting the MT1-MMP-mediated MMP2 activation. (2) It was studied in other cancers ${ }^{23,24}$ that SPOCK1 can regulate the expression and activation of MT1-MMP and MMP2 by regulating signaling pathway or epithelial-mesenchymal transition (EMT). The SPOCK2 may inhibit the expression and activation of MT1-MMP and MMP2 by regulating signaling pathways or EMT in the EC. The mechanism of the inhibiting MMP2 by SPOCK2 in the EC deserves to be further explored.

In conclusion, this study suggests that SPOCK2 may be related to the progression of EC by regulating the biological 
behavior of cancer cells, achieved partly through regulating the protein expression of MT1-MMP and MMP2 and the activation of MMP2, and SPOCK2 may be a potential therapeutic target for the treatment of EC. However, further evidence of how SPOCK2 regulates MT1-MMP and MMP2 protein expression and whether there is any other pathway involved in SPOCK2 function requires further investigation.

\section{Declaration of Conflicting Interests}

The authors declared no potential conflicts of interest with respect to the research, authorship, and/or publication of this article.

\section{Funding}

The author(s) disclosed receipt of the following financial support for the research, authorship, and/or publication of this article: The present study was supported by grants from the National Natural Science Foundation of China (No. 81501235).

\section{References}

1. Oki S, Sone K, Oda K, et al. Oncogenic histone methyltransferase EZH2: a novel prognostic marker with therapeutic potential in endometrial cancer. Oncotarget. 2017;8(25):40402-40411.

2. Bokhman JV. Two pathogenetic types of endometrial carcinoma. Gynecol Oncol. 1983;15(1):10-17.

3. Noer MC, Antonsen SL, Ottesen B, Christensen IJ, Høgdall C. Type I versus type II endometrial cancer: differential impact of comorbidity. Int J Gynecol Cancer. 2018;28(3):586-593.

4. Nordgard SH, Johansen FE, Alnaes GI, et al. Genome-wide analysis identifies $16 \mathrm{q}$ deletion associated with survival, molecular subtypes, mRNA expression, and germline haplotypes in breast cancer patients. Genes Chromosomes Cancer. 2008;47(8):680-696.

5. Chung W, Kwabi-Addo B, Ittmann M, et al. Identification of novel tumor markers in prostate, colon and breast cancer by unbiased methylation profiling. PLoS One. 2008;3(4):e2079.

6. Ren F, Wang DB, Li T, Chen YH, Li Y. Identification of differentially methylated genes in the malignant transformation of ovarian endometriosis. J Ovarian Res. 2014;7:73.

7. Wang CT, Wang DB, Liu KR, et al. Inducing malignant transformation of endometriosis in rats by long-term sustaining hyperestrogenemia and type II diabetes. Cancer Sci. 2015;106(1):43-50.

8. Rodolakis A, Thomakos N, Akrivos N, et al. Clinicopathologic insight of simultaneously detected primary endometrial and ovarian carcinomas. Arch Gynecol Obstet. 2012;285(3):817-821.

9. Alliel PM, Perin JP, Jolles P, Bonnet F. Testican, a multidomain testicular proteoglycan resembling modulators of cell social behaviour. Eur J Biochem. 1993;214(1):347-350.

10. Sambuudash O, Kim HS, Cho MY. Lack of aberrant methylation in an adjacent area of left-sided colorectal cancer. Yonsei Med J. 2017;58(4):749-755.

11. Ren F, Wang DB, Li T. Epigenetic inactivation of SPOCK2 in the malignant transformation of ovarian endometriosis. Zhonghua Fu Chan Ke Za Zhi. 2011;46(11):822-825.
12. Song H, Pan D, Sun W, et al. SiRNA directed against annexin II receptor inhibits angiogenesis via suppressing MMP2 and MMP9 expression. Cell Physiol Biochem. 2015;35(3):875-884.

13. Wang HY, Tu YS, Long J, et al. SRF-miR 29b-MMP2 axis inhibits NSCLC invasion and metastasis. Int J Oncol. 2015;47(2): 641-649.

14. Yi SA, Ryu HW, Lee DH, Han JW, Kwon SH. HP1 $\beta$ suppresses metastasis of human cancer cells by decreasing the expression and activation of MMP2. Int J Oncol. 2014;45(6):2541-2548.

15. Sato H, Takino T. Coordinate action of membrane-type matrix metalloproteinase-1 (MT1-MMP) and MMP-2 enhances pericellular proteolysis and invasion. Cancer Sci. 2010;101(4): 843-847.

16. Al-Raawi D, Abu-El-Zahab H, El-Shinawi M, Mohamed MM. Membrane type-1 matrix metalloproteinase (MT1-MMP) correlates with the expression and activation of matrix metalloproteinase-2 (MMP-2) in inflammatory breast cancer. Int J Clin Exp Med. 2011;4(4):265-275.

17. Koshikawa N, Giannelli G, Cirulli V, Miyazaki K, Quaranta V. Role of cell surface metalloprotease MT1-MMP in epithelial cell migration over laminin-5. J Cell Biol. 2000;148(3): 615-624.

18. Lehti K, Lohi J, Juntunen MM, Pei D, Keski-Oja J. Oligomerization through hemopexin and cytoplasmic domains regulates the activity and turnover of membrane-type 1 matrix metalloproteinase. J Biol Chem. 2002;277(10):8440-8448.

19. Nakada M, Yamada A, Takino T, et al. Suppression of membrane-type 1 matrix metalloproteinase (MMP)-mediated MMP-2 activation and tumor invasion by testican 3 and its splicing variant gene product, N-Tes. Cancer Res. 2001; 61(24):8896-8902.

20. Nakada M, Miyamori H, Yamashita J, Sato H. Testican 2 abrogates inhibition of membrane-type matrix metalloproteinases by other testican family proteins. Cancer Res. 2003;63(12): 3364-3369.

21. Berger EA, McClellan SA, Barrett RP, Hazlett LD. Testican-1 promotes resistance against Pseudomonas aeruginosa-induced keratitis through regulation of MMP-2 expression and activation. Invest Ophthalmol Vis Sci. 2011;52(8):5339-5346.

22. Kudo T, Takino T, Miyamori H, Thompson EW, Sato H. Substrate choice of membrane-type matrix metalloproteinase is dictated by tissue inhibitor of metalloproteinase-2 levels. Cancer Sci. 2007;98(4):563-568.

23. Yang J, Yang Q, Yu J, Li X, Yu S, Zhang X. SPOCK1 promotes the proliferation, migration and invasion of glioma through PI3K/ AKT and Wnt/ $\beta$-catenin signaling pathways. Oncol Rep. 2016; 35(6):3566-3576.

24. Chen D, Zhou H, Liu G, Zhao Y, Cao G, Liu Q. SPOCK1 promotes the invasion and metastasis of gastric cancer through Sluginduced epithelial-mesenchymal transition. J Cell Mol Med. 2018;22(2):797-807. 